\title{
Aberrant Expression of Epithelial-Mesenchymal Transition Markers in Early Gastric Cancer: Clinical Application
}

\author{
Moon Kyung Joo \\ Division of Gastroenterology, Department of Internal Medicine, Korea University College of Medicine Guro Hospital, Seoul, Korea
}

See "Immunohistochemical Expression of Epithelial-Mesenchymal Transition Markers in Early Gastric Cancer: Cancer Tissue versus Noncancer Tissue" by Hee Jae Jung, Su Jin Hong, Shin Hee Kim, on page 464-471.

Gastric cancer is one of the frequently diagnosed cancers worldwide, and the third leading cause of cancer-related death. ${ }^{1}$ Early detection and complete removal of the tumor are effective therapeutic strategies in the treatment of gastric cancer. Similar to other cancers, the prognosis of gastric cancer remains generally poor when diagnosed in the advanced stages. Endoscopic submucosal dissection (ESD) is currently established as one of the optimal treatment modalities for early gastric cancer (EGC). Further, EGC with submucosal invasion or undifferentiated type histology is considered as an expanded indication for ESD. ${ }^{2}$ Nonetheless, endoscopists need to exercise caution while selecting candidates with suspected submucosal invasion or undifferentiated type histology for ESD of EGC before the procedure, and in such cases, non-curative resection by histopathologic evaluation is often observed after the procedure. ${ }^{3,4}$ Hence, clinical practitioners need to be cautious and aware of the various factors that promote the invasiveness of EGCs.

As seen in any other type of cancer, epithelia-mesenchymal transition (EMT) is a crucial step towards the initiation and progression of gastric cancer. Expression of mesenchymal

Received: July 16, 2019 Accepted: August 9, 2019

Correspondence: Moon Kyung Joo

Division of Gastroenterology, Department of Internal Medicine, Korea University College of Medicine Guro Hospital, 148 Gurodong-ro, Guro-gu, Seoul 08308, Korea

Tel: +82-2-2626-3007, Fax: +82-2-2626-1038, E-mail: latyrx@korea.ac.kr ORCID: https://orcid.org/0000-0001-6050-3695

(cc) This is an Open Access article distributed under the terms of the Creative Commons Attribution Non-Commercial License (http://creativecommons.org/ licenses/by-nc/3.0) which permits unrestricted non-commercial use, distribution, and reproduction in any medium, provided the original work is properly cited. cell markers such as Snaill and vimentin promotes loss of epithelial cell contacts, gain of mesenchymal phenotype, and cellular migration and invasion of gastric cancer cells. ${ }^{5}$ E-cadherin is a typical epithelial marker in gastric epithelial cells and downregulation of E-cadherin expression is a hallmark of diffuse-type gastric cancer. ${ }^{6}$ However, aberrant expression of EMT markers in gastric cancer tissue has been predominantly evaluated in advanced gastric cancer and rarely reported in EGCs. In this translational study, Jung et al. investigated the differential expression of EMT markers between EGC and non-tumorous gastric tissues. ${ }^{7}$ Authors chose transforming growth factor (TGF)- $\beta$, vascular endothelial growth factor (VEGF), $\alpha$-smooth muscle actin ( $\alpha$-SMA), and vimentin as mesenchymal markers, and E-cadherin as an epithelial marker, and investigated their expression level in cancer and non-tumor tissues by immunohistochemistry (IHC). Interestingly, they separately classified non-tumorous tissues as "noncancer tissue", which was defined as tissues located $5 \mathrm{~cm}$ outside the tumor margin, and "NONCANCER TISSUE" as tissue which was obtained at post-ESD scar 3 months after ESD. The expression levels of the mesenchymal markers (TGF- $\beta$, VEGF, $\alpha$-SMA, and vimentin) were significantly higher in cancer tissue than in noncancer tissue as well as "NONCANCER TISSUE"; however, the expression of E-cadherin did not significantly differ between cancer and non-tumor gastric tissues. This study is significant as it reveals the differential expression of mesenchymal markers in EGC tissues resected by ESD, and indicates that several mesenchymal markers have a potential to serve as surrogate markers for complete remission of the tumor at the resection site after curative ESD. The reason for 
the lack of difference in the E-cadherin expression levels between cancerous and non-tumor tissues could be associated with the limited number of specimens analyzed with poorly differentiated histology ( $n=2$ among total 19 patients). Further, loss of E-cadherin is more prominent in gastric cancers with undifferentiated type histology.

This study, however, has several limitations. Firstly, owing to the limited number of specimens $(n=19)$, the authors could not illustrate the aberrant expression of EMT markers according to the differentiation and invasion depth (mucosa vs. submucosa). It might be interesting if the authors could further show the differential expression of EMT markers between T1a and T1b cancers, which would be clinically attractive and useful in terms of the appropriate selection of patients for ESD. Next, authors could only show EMT marker expression using IHC stain. However, alteration of EMT marker expression usually accompanies significant changes in cellular morphology: from epithelial phenotype characterized by round and epithelioid-like appearance to a mesenchymal phenotype, with sharp, elongated, and fibroblast-like shape. ${ }^{9}$ Such morphological changes could be easily distinguished if immunofluorescence staining was performed.

Like other fields of medicine, treatment of gastric cancer in the future should focus on "precision medicine", which analyzes the molecular characteristics of individual gastric cancer patients and devise optimal treatment strategies. In the field of endoscopic treatment of EGC, such attempts are expected to be more active. Hopefully, in the future, the number of therapeutic endoscopists who emphasize on the comprehensive analysis of a wide range of biologic and molecular characteris- tics of EGC will increase.

Conflicts of Interest

The author has no financial conflicts of interest.

\section{REFERENCES}

1. Herrero R, Park JY, Forman D. The fight against gastric cancer - the IARC Working Group report. Best Pract Res Clin Gastroenterol 2014;28:1107-1114

2. Gotoda T, Yanagisawa A, Sasako M, et al. Incidence of lymph node metastasis from early gastric cancer: estimation with a large number of cases at two large centers. Gastric Cancer 2000;3:219-225.

3. Ono H, Yao K, Fujishiro M, et al. Guidelines for endoscopic submucosal dissection and endoscopic mucosal resection for early gastric cancer. Dig Endosc 2016;28:3-15.

4. Bang CS, Park JM, Baik GH, et al. Therapeutic outcomes of endoscopic resection of early gastric cancer with undifferentiated-type histology: a Korean ESD registry database analysis. Clin Endosc 2017;50:569-577.

5. Peng Z, Wang CX, Fang EH, Wang GB, Tong Q. Role of epithelial-mesenchymal transition in gastric cancer initiation and progression. World J Gastroenterol 2014;20:5403-5410.

6. Cho SY, Park JW, Liu Y, et al. Sporadic early-onset diffuse gastric cancers have high frequency of somatic $\mathrm{CDH} 1$ alterations, but low frequency of somatic RHOA mutations compared with late-onset cancers. Gastroenterology 2017;153:536-549.e26.

7. Jung HJ, Hong SJ, Kim SH. Immunohistochemical expression of epithelial-mesenchymal transition markers in early gastric cancer: cancer tissue versus noncancer tissue. Clin Endosc 2019;52:464-471.

8. Chen HC, Chu RY, Hsu PN, et al. Loss of E-cadherin expression correlates with poor differentiation and invasion into adjacent organs in gastric adenocarcinomas. Cancer Lett 2003;201:97-106.

9. Kim SH, Yoo HS, Joo MK, et al. Arsenic trioxide attenuates STAT-3 activity and epithelial-mesenchymal transition through induction of SHP1 in gastric cancer cells. BMC Cancer 2018;18:150. 